\title{
Análisis de los convenios de cooperación para la conservación de Los Pantanos de Villa (Lima, Perú)
}

Analysis of cooperation agreements for the conservation of Los Pantanos de Villa (Lima, Peru)

\author{
Marycielo Miranda ${ }^{1 *}{ }^{\oplus}$ y Juana Basto ${ }^{2} \odot$
}

1 Autoridad Municipal de los Pantanos de Villa (Prohvilla). Lima, Perú. Pasante de la Oficina General de Asesoría Jurídica en PROHVILLA 2020-II.

2 Autoridad Municipal de los Pantanos de Villa (Prohvilla). Lima, Perú. Pasante de la Oficina General de Asesoría Jurídica en PROHVILLA 2020-II.

\section{SOUTH} SUSTAINABILITY

Citar como: Miranda M. y Basto J. (2021). «Análisis de los convenios de cooperación para la conservación de Los Pantanos de Villa (Lima, Perú)». South Sustainability, 2(2), e041. DOI: 10.21142/SS-0202-2021-pb003

\section{Artículo recibido: 5/10/2021} Arbitrado por pares

Artículo aceptado: 31/12/2021

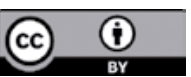

(c) Las autoras, 2021. Publicado por la Universidad Científica del Sur (Lima, Perú)

${ }^{*}$ E-mail de correspondencia: marycielo.mirandac@gmail.com

\begin{abstract}
RESUMEN
La suscripción de convenios es importante para fortalecer la gestión de la conservación de las áreas naturales. En el presente artículo se analiza el estado actual de los convenios de cooperación suscritos por la Autoridad Municipal de los Pantanos de Villa (Prohvilla, encargada de la gestión de la Zona de Reglamentación Especial, que comprende el Humedal Pantanos de Villa) entre 2019 y 2020. Para ello, se revisaron los convenios que se encuentran en su registro y se entrevistó a los responsables de Prohvilla. El registro contó con 22 convenios para ese periodo de estudio, que en su mayoría han sido firmados con personas naturales (59\%), son de tipo promocional (68\%), están activos (77\%) y han brindado beneficios para la gestión del área (73\%). A partir de lo encontrado, se sugiere que se establezcan mesas de trabajo, planes de seguimiento y estandarización de las propuestas, de manera que sean más efectivos el seguimiento y la ejecución de estos convenios.
\end{abstract}

Palabras clave: conservación, cooperación, convenios, humedales, Prohvilla ABSTRACT

The signing of agreements is important in order to strengthen the conservation management of natural areas such as wetlands. In this article, the current status of the cooperation agreements signed by PROHVILLA (responsible for the management of Los Pantanos de Villa wetland) between 2019 and 2020 is analyzed. To this end, the agreements contained in its records were reviewed and those in charge of PROHVILLA were interviewed. The records contained 22 agreements dating from the study period, the majority of which (59\%) were signed with natural persons, were of a promotional nature $(68 \%)$, remain active $(77 \%)$, and have contributed benefits for the management of the area $(73 \%)$. Based on these findings, it is suggested that work groups, monitoring plans and the standardization of proposals be established, in order to ensure greater effectiveness in the implementation and monitoring of agreements.

Keywords: conservation, cooperation, agreements, wetlands, PROHVILLA 


\section{Introducción}

Los humedales son áreas cuya capa freática se encuentra en la superficie terrestre o donde la tierra está cubierta por aguas; es decir, es una zona que permanece en condiciones de inundación, con la cual se logra generar un ecosistema (Secretaría de la Convención de Ramsar, s. f.). La misma Secretaría reconoce la importancia de estas áreas, dado que aportan una gran diversidad biológica, por lo que la gestión que realiza Prohvilla, mediante sus diversos instrumentos de administración, debe ser orientada con un enfoque de desarrollo sostenible. Por tanto, los convenios de cooperación contribuyen a la conservación del área natural, debido a que su objetivo principal es promover y desarrollar proyectos y acciones en beneficio de la preservación del Humedal Pantanos de Villa (HPV).

Prohvilla es un organismo público descentralizado de la Municipalidad Metropolitana de Lima, y se encarga de la gestión ambiental y la administración de la Zona de Reglamentación Especial de Los Pantanos de Villa (Ordenanza 2264-MML). Esta Zona de Reglamentación Especial comprende el HPV, así como el entorno territorial que ejerce influencia sobre sus procesos ecológicos inherentes. El HPV es un lugar reconocido como sitio Ramsar 884 por la Convención de Humedales del mismo nombre, al ser una zona de alimentación, reproducción, albergue, descanso y refugio de aves residentes y migratorias del continente americano. Así también, el HPV es un área natural protegida con la categoría de refugio de vida silvestre que integra el Sistema Nacional de Áreas Naturales Protegidas por el Estado, según lo señalado en la misma ordenanza. De ahí que resulte vital que Prohvilla, en el marco de su competencia, deba promover la conservación y el desarrollo sostenible del HPV, no solo por los servicios ecosistémicos que brinda, sino también por los efectos del cambio climático que permite contrarrestar (León, 2020). La conservación se constituye en su finalidad institucional.

Es así que, con el propósito de cumplir con la finalidad institucional, Prohvilla puede establecer estrategias que permitan su cumplimiento. En atención a ello, el estatuto y el reglamento de Prohvilla reconocen que la entidad en mención puede suscribir convenios de cooperación con entidades públicas y privadas para la ejecución de actividades en el área natural y la zona de reglamentación especial, así como celebrar todos los actos y contratos necesarios para el cumplimiento de sus objetivos.

Esto último encuentra sustento, además, en los artículos 87 y 88 del Texto Único Ordenado de la Ley 27444, Ley de Procedimiento Administrativo General, en los que se reconoce que las entidades públicas pueden establecer relaciones de colaboración entre sí o con instituciones del sector privado (tercero o contraparte firmante, en adelante), toda vez que permitan el cumplimiento de las finalidades de las respectivas entidades públicas, se encuentren en el ámbito de su respectiva competencia y no se vulneren normas de orden público. Estas relaciones de colaboración pueden materializarse en convenios de colaboración u otros medios legalmente admisibles. Según anota Tirado (2011), el uso de los convenios como medio de cooperación permite una interacción activa entre las entidades vinculadas, lo que genera intercambio de información, beneficios y trabajo conjunto para alcanzar los objetivos establecidos.

Los convenios de colaboración, también denominados convenios de la administración pública o de cooperación, son acuerdos voluntarios y vinculantes entre una entidad pública y el tercero, en los cuales se busca satisfacer necesidades de ambas partes, pero que coinciden necesariamente con el interés general (Santos, 2009). Por su parte, la directiva «Normas y procedimientos sobre convenios de cooperación y otros documentos de cooperación que suscriba la Municipalidad Metropolitana de Lima», aprobada mediante Resolución de Gerencia Municipal Metropolitana 054-2020-MML-GMM, ha definido a los convenios de cooperación como aquellos acuerdos suscritos por la entidad pública con una o más entidades, públicas o privadas, nacionales o extranjeras, instituciones u organismos internacionales, orientados a la realización de actividades de manera conjunta y de interés común en el marco de las funciones y competencias de aquella entidad. Además, los clasificó en dos tipos, según su naturaleza: por un lado, los convenios de cooperación marco, que son acuerdos propuestos para el logro de un conjunto de objetivos generales y de acciones de interés común con un plazo de vigencia determinado por las partes; y, por otro lado, los convenios de cooperación específicos, que son acuerdos realizados para el desarrollo de acciones específicas de un fin determinado con plazo de vigencias igualmente determinado por las partes, previo sustento técnico.

En línea a lo expresado y en el marco de la competencia Prohvilla, la entidad ha venido suscribiendo convenios de cooperación con distintas entidades públicas y privadas. Desde 2019 a la fecha, según información proporcionada por la Oficina General de Asesoría Jurídica (OGAJ) para efectos del presente artículo, Prohvilla ha gestionado 22 convenios de cooperación: 16 efectivamente suscritos; 3 aprobados por el Consejo Directivo, pero sin la firma de las partes vinculadas; y 3 con evaluación favorable de la Dirección Técnica, que serán puestos para discusión y votación del Consejo Directivo de Prohvilla, los cuales serán considerados como convenios de cooperación suscritos para efectos del presente artículo.

En ese sentido, en el presente policy brief (o resumen de política, por su traducción en español) de tipo objetivo, se analizan los convenios de cooperación suscritos por Prohvilla de 2019 a 2020 y se brindan recomendaciones a fin de promover la suscripción de convenios de cooperación en Prohvilla. 


\section{Enfoques y discusión}

Para la presente investigación se realizaron entrevistas personales a los responsables de los Órganos de Línea, del Área de Comunicaciones, Educación Ambiental y Participación Ciudadana, y de la Dirección Técnica de Prohvilla, entre el 1 y el 30 de septiembre de 2020.

A partir de las entrevistas realizadas, se estableció que Prohvilla ha suscrito 22 convenios de cooperación, que fueron sistematizados en variables, como se muestra en la figura 1.

En primer lugar, en la figura 1.a) se observa que más de la mitad de los convenios de cooperación (59\%) fueron suscritos con personas naturales, mientras que los demás se firmaron con personas jurídicas.

En segundo lugar, en la figura 1.b) se advierte que la mayoría de los convenios comprenden el tipo de beneficio promocional a favor de Prohvilla (68\%), a partir del cual se busca visibilizar el HPV de la Zona de Reglamentación Especial de Los Pantanos de Villa como un espacio de necesaria conservación, de conformidad con lo mencionado en el apartado anterior.

Sin perjuicio de la predominancia del tipo de beneficio promocional en los convenios de cooperación suscritos por Prohvilla, es posible observar que, aunque en menores porcentajes, Prohvilla también ha suscrito convenios con beneficios académicos (9\%), científico-formativos (14\%) y de gestión de talento de su personal (5\%), los cuales buscan generar información e incentivar una adecuada actuación de las personas vinculadas para la conservación del HPV; y con beneficios de conservación (4 \%), que permiten el cumplimiento directo de la finalidad institucional a diferencia de los otros.
En tercer lugar, en la figura 1.c) se visualiza que, a la fecha, más de las tres cuartas partes de los convenios suscritos por Prohvilla están activos (77\%); es decir, que se vienen realizando actividades en el marco del beneficio comprometido a favor de Prohvilla.

Y, en cuarto y último lugar, en la figura 1.d) se indica que la mayoría de los convenios de cooperación suscritos por Prohvilla son efectivamente beneficiosos para la entidad (73\%), y con ello se permite coadyuvar al cumplimiento de la finalidad de conservación del HPV de la entidad.

Los beneficios señalados previamente han sido informados por los responsables de los Órganos de Línea, del Área de Comunicaciones, Educación Ambiental y Participación Ciudadana, y de la Dirección Técnica de Prohvilla, durante las entrevistas personales, y efectivamente corroborados por las autoras a través de la revisión de informes institucionales y materiales visuales, con apoyo de la OGAJ de la misma entidad.

En ese sentido, se puede señalar que, en su mayoría, los convenios han sido firmados con personas naturales (59 \%), son de tipo promocional (68\%), se encuentran activos (77\%) y han brindado beneficios para la gestión del área (73\%), todo lo cual contribuye con la finalidad institucional de conservación del HPV.

\section{Conclusiones y recomendaciones}

El análisis de los 22 convenios muestra el importante rol de aquellos firmados con personas naturales y de tipo promocional. Asimismo, muestra que un gran porcentaje de los convenios se encuentran activos y han generado beneficios para las actividades de gestión que realiza Prohvilla.

B.

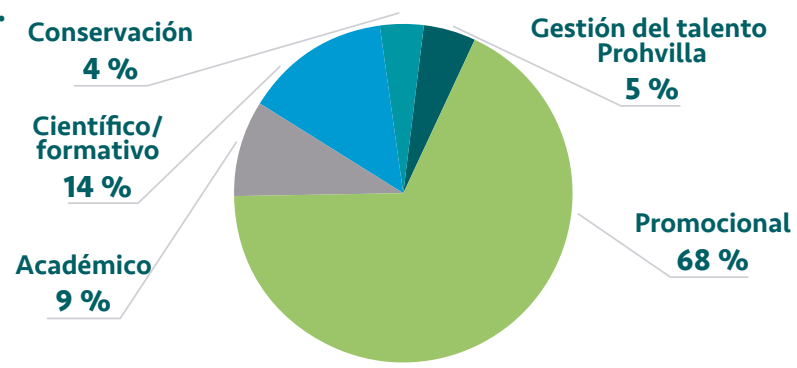

D.

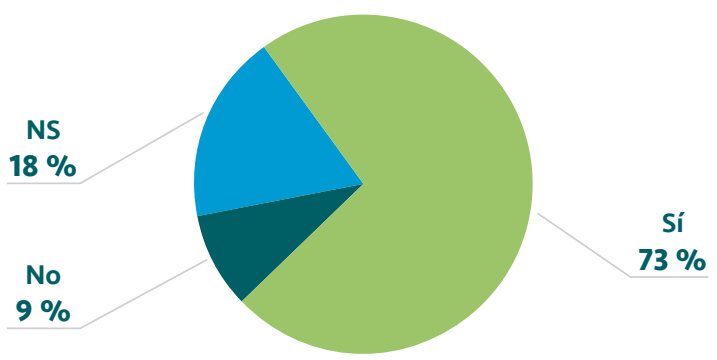

Figura 1. Distribución de los convenios de cooperación de Prohvilla, en función: a) del tipo de institución contraparte firmante; b) del tipo de beneficio comprometido a favor de Prohvilla; c) del estado actual para Prohvilla; y d) del beneficio para Prohvilla. 
A fin de promover la suscripción de convenios de cooperación en Prohvilla, y sobre la base de lo comentado por los responsables durante las entrevistas, se recomienda establecer mesas de trabajo antes de la propuesta del convenio, que ayuden a identificar y atender todas las necesidades con una visión integral. Asimismo, para verificar los beneficios de los convenios suscritos en el tiempo, se debería realizar una matriz de seguimiento con plazos previamente determinados. Y, finalmente, con el propósito de facilitar la suscripción de más convenios de cooperación y seguir un orden en el proceso de suscripción de dichos convenios, se recomienda que la entidad a pruebe una directiva con formatos de convenios previamente establecidos cuando se trate de fines similares, replicando así el instrumento de gestión que emplean distintas entidades públicas para la suscripción de convenios de cooperación, como la Municipalidad Metropolitana de Lima, el Sistema Nacional de Evaluación, Acreditación y Certificación de la Calidad Educativa (Sineace), el Consejo Nacional de Ciencia, Tecnología e Innovación Tecnológica (Concytec), entre otras.

\section{Agradecimientos}

A la Autoridad Municipal de los Pantanos de Villa (Prohvilla), por la oportunidad de participar en el programa de pasantía 2020-II de la entidad.

A Yesabell Ostos y Marlon Hurtado, jefa y asistente legal de la Oficina General de Asesoría Jurídica de Prohvilla, respectivamente, por su constante apoyo en el desarrollo del presente artículo.

Al doctor Héctor Aponte, investigador asociado de investigación en la Universidad Científica del Sur (Lima, Perú), por sus comentarios al presente artículo.

A los responsables de las Oficinas y Dirección Técnica de Prohvilla, por las entrevistas personales brindadas con propósitos de recolección de datos para el presente artículo.

\section{Fuente de financiamiento}

Autofinanciado y en colaboración con Prohvilla.

\section{Potenciales conflictos de interés}

Las autoras declaran no tener conflicto de intereses.

\section{Referencias bibliográficas}

León Sulca, G. (2020). «Gobernanza ambiental y conservación: las gestiones del SERNANP y PROHVILLA en el Refugio de Vida Silvestre Los Pantanos de Villa». Revista Argumentos, 1(1), pp. 119-124. DOI: 10.46476/ra.vi1.20.

Martin Tirado, R. J. (2011). «Reformas a los mecanismos de colaboración administrativa a propósito de los diez años de vigencia de la ley 27444». Derecho PUCP, 67, pp. 309-327. Disponible en: http:// revistas.pucp.edu.pe/index.php/derechopucp/article/view/2948

Municipalidad Metropolitana de Lima. (2020). Resolución de Gerencia Municipal Metropolitana N. ${ }^{\circ}$ 054-2020-MML-GMM.

Santos Rodríguez, J. E. (2009). «Consideración sobre los contratos y convenios interadministrativos». Revista Digital de Derecho Administrativo, (1), pp. 1-24. Disponible en: https://revistas. uexternado.edu.co/index.php/Deradm/article/view/2592

Secretaria de la Convención de Ramsar. (S. f.). «¿Qué son los humedales?». Documento informativo Ramsar No. 1. Disponible en: https://www.ramsar.org/sites/default/files/documents/library/ info2007sp-01.pdf 